\title{
Betreuung von Frauen mit Rückenmarkschädigung
}

Eine Frau im Rollstuhl beim Mutterwerden und in der ersten Zeit mit dem Baby zu begleiten, ist für viele Hebammen eine neue Erfahrung. 2018 erschien die S2k-Leitlinie: Schwangerschaft, Geburt und Wochenbett bei Frauen mit Querschnittlähmung. Sie beinhaltet interdisziplinäre Standards und praxisorientierte Hilfen zur Betreuung und Beratung von Frauen mit Kinderwunsch bzw.Schwangerschaft bei Rückenmarkschädigung.

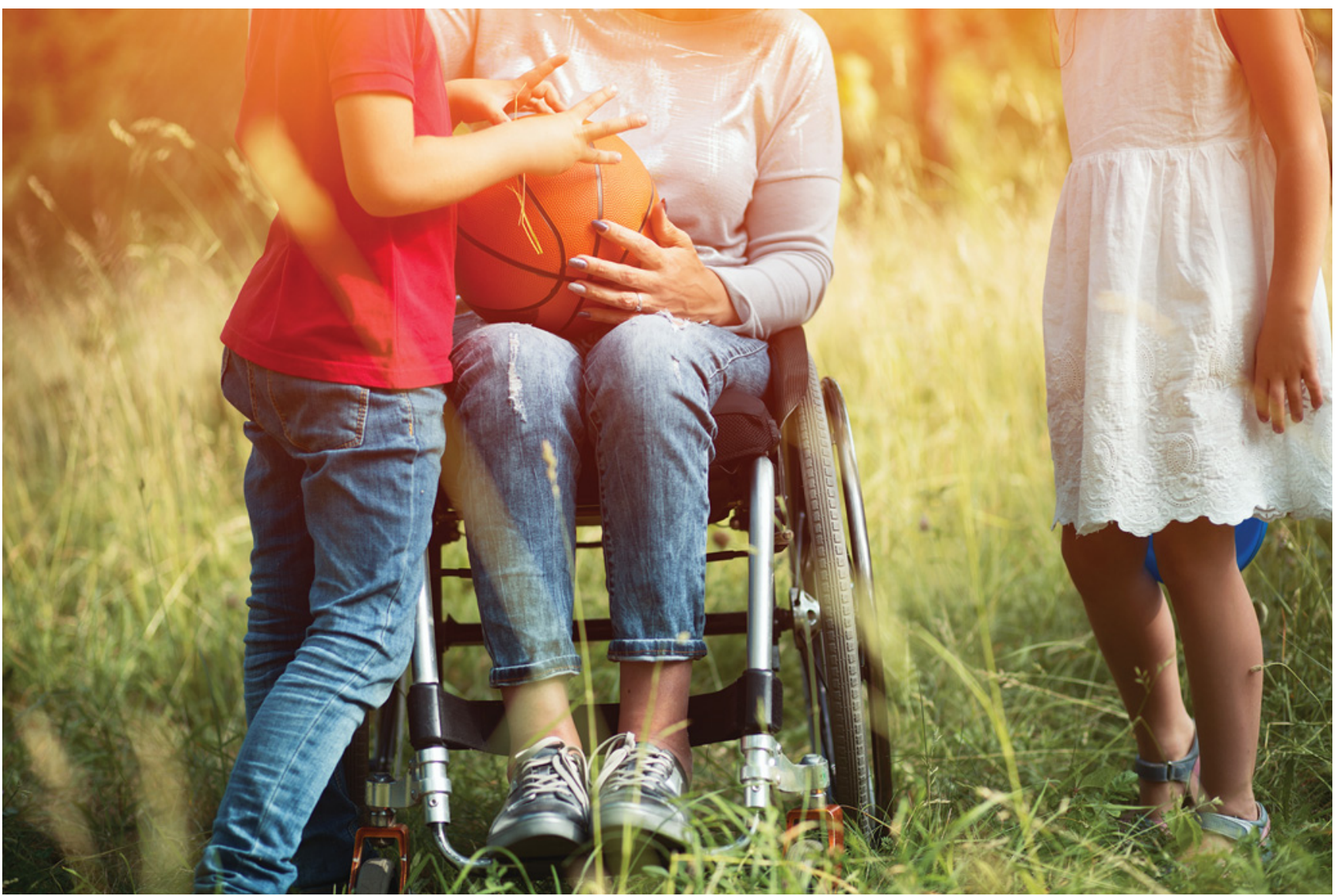

Die Schwerpunktbeiträge dieser Ausgabe beleuchten die Betreuung von Schwangeren, Gebärenden und jungen Müttern mit Handicap aus verschiedenen Perspektiven. Dr. med. Ines Kurze vermittelt in ihrem Übersichtsartikel Ursachen, Symptome und Klassifikation des Querschnittsyndroms und stellt den ganzheitlichen Behandlungsansatz am Querschnittgelähmten-Zentrum in Bad Berka vor. Der zweite Artikel fasst praxisrelevante Aspekte der neuen Leitlinie zusammen. Und schließlich berichten Mütter im Rollstuhl, wie sie Schwangerschaft, Geburt und Wochenbett erlebten und ihr Leben mit Baby meistern - Kontaktadressen, Ausstattungstipps und Links zum Weiterlesen inklusive. (Foto: Svyatoslav Lypynskyy - stock.adobe.com; Symbolbild)

- Paraplegiologie - Wissen und Behandlungsansatz für das Querschnittsyndrom

- Schwangerschaft, Geburt und Wochenbett bei Frauen mit Querschnittlähmung
- Wie Frauen im Rollstuhl Schwangerschaft, Geburt und Mutterrolle meistern 


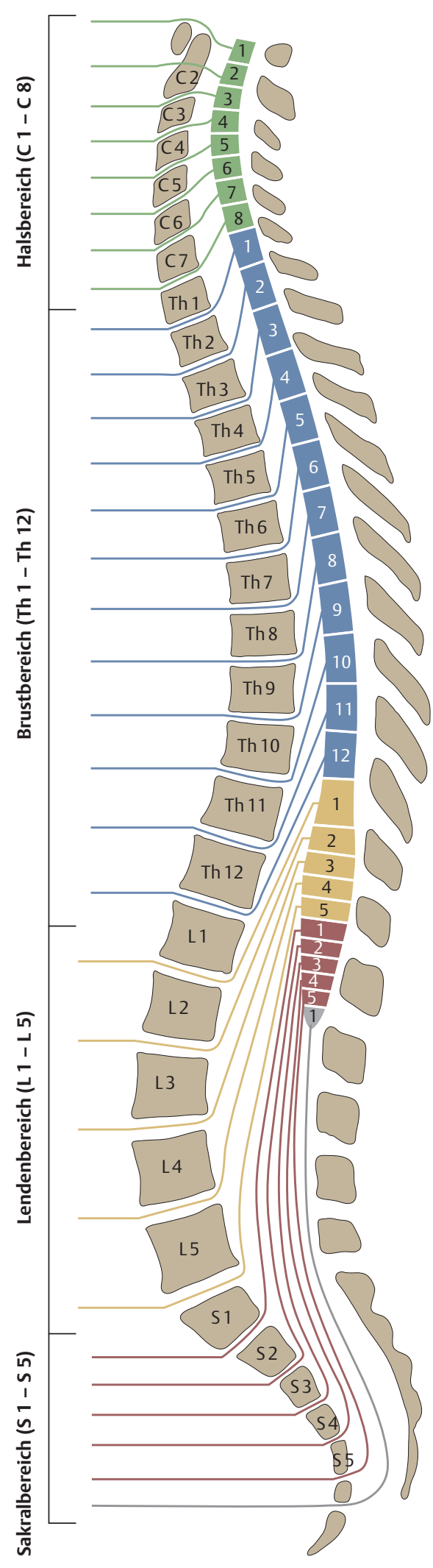

\begin{tabular}{|c|c|c|}
\hline $\begin{array}{l}\text { Läsionshöhe } \\
\text { (Kennmuskeln) }\end{array}$ & $\begin{array}{l}\text { Funktionelle Fähigkeiten } \\
\text { a) Körperpflege } \\
\text { b) Handfunktion } \\
\text { c) Fortbewegungsmöglichkeit }\end{array}$ & Hilfsmittel \\
\hline $\begin{array}{l}\text { C } 3 / 4 \\
\text { (Diaphragma) }\end{array}$ & $\begin{array}{l}\text { a) vollständig pflegeabhängig } \\
\text { c) Elektrorollstuhl }\end{array}$ & $\begin{array}{l}\text { - mech. Rollstuhl (RST) } \\
\text { - Elektrorollstuhl } \\
\text { - Lifter } \\
\text { - Pflegebett mit Steh- } \\
\text { vorrichtung } \\
\text { - Atemtherapiegerät }\end{array}$ \\
\hline $\begin{array}{l}\text { C } 4 / 5 \\
\text { (M. biceps brachii) }\end{array}$ & $\begin{array}{l}\text { a) vorwiegend pflegeabhängig } \\
\text { b) Essen, Schreiben mit spez. } \\
\text { Hilfsmitteln bedingt möglich } \\
\text { c) mech. RST auf ebenem } \\
\text { Boden für kurze Strecken, } \\
\text { geschickter Umgang mit } \\
\text { dem Elektrorollstuhl }\end{array}$ & $\begin{array}{l}\text { - mech. u. Elektro-RST } \\
\text { (Handsteuerung) } \\
\text { - Lifter } \\
\text { - Pflegebett, s. o. } \\
\text { - Hilfen für die bedingte } \\
\text { Handfunktion } \\
\text { - evtl. Atemtherapie- } \\
\text { gerät }\end{array}$ \\
\hline $\begin{array}{l}\text { C } 5 / 6 \\
\text { (M. ext. carp. rad.) }\end{array}$ & $\begin{array}{l}\text { a) teilweise pflegeunabhängig } \\
\text { b) bei gut ausgebildeter } \\
\text { „aktiver“ Funktionshand } \\
\text { Essen und Schreiben } \\
\text { möglich } \\
\text { c) mech. RST auf ebenen } \\
\text { Strecken möglich, evtl. } \\
\text { Bedienung eines } \\
\text { adaptierten Pkws }\end{array}$ & $\begin{array}{l}\text { - mech. (evtl. Elektro-) } \\
\text { RST } \\
\text { - ggf. Lifter } \\
\text { - Pflegebett, s. o. } \\
\text { - Hilfen für die Hand- } \\
\quad \text { funktion } \\
\text { - adaptierter Pkw }\end{array}$ \\
\hline $\begin{array}{l}\text { C } 6 / 7 \\
\text { (M. triceps brachii) }\end{array}$ & $\begin{array}{l}\text { a) teilweise selbstständig } \\
\text { b) gute „Funktionshand“ } \\
\text { c) mech. RST, auch in } \\
\text { unebenem Gelände, } \\
\text { adaptierter Pkw }\end{array}$ & $\begin{array}{l}\text { - mech. RST } \\
\text { - Funktionshilfen, s. o. } \\
\text { - Pflegebett, s. o. } \\
\text { ggf. mech. Stehgerät } \\
\text { - adaptierter Pkw }\end{array}$ \\
\hline $\begin{array}{l}\text { C } 7 / 8 \\
\text { (Mm. dig. flex. } \\
\text { Mm. dig. ext. } \\
\text { M. lat. dors.) }\end{array}$ & $\begin{array}{l}\text { a) selbstständig } \\
\text { b) bedingte Fingerteilfunktion } \\
\text { c) gute RST-Fertigkeiten } \\
\text { adaptierter Pkw }\end{array}$ & $\begin{array}{l}\text { - mech. RST } \\
\text { - mech. Stehgerät, evtl. } \\
\quad \text { Aufrichte-RST } \\
\text { - adaptierter Pkw }\end{array}$ \\
\hline $\begin{array}{l}\text { Th } 1-9 \\
\text { (Mm. intercostales } \\
\text { Mm. abdominales) } \\
\text { (teilweise) }\end{array}$ & c) gute RST-Fertigkeit & $\begin{array}{l}\text { - mech. RST } \\
\text { - mech. Stehgerät, evtl. } \\
\quad \text { Aufrichte-RST } \\
\text { - adaptierter Pkw }\end{array}$ \\
\hline $\begin{array}{l}\text { Th } 10-\mathrm{L} 2 \\
\text { (Rumpfmuskeln, } \\
\text { Hüftbeuger) }\end{array}$ & c) rollstuhlabhängig & $\begin{array}{l}\text { - mech. RST } \\
\text { - Stützapparate für das } \\
\text { Steh- bzw. Gehtraining } \\
\text { - Barren, Unterarm- } \\
\text { stützen } \\
\text { - adaptierter Pkw }\end{array}$ \\
\hline $\begin{array}{l}\text { L } 3 / 4 \\
\text { (M. quadriceps } \\
\text { M. tib. ant. } \\
\text { M. semitendinosus } \\
\text { M. semimem- } \\
\text { branosus }\end{array}$ & $\begin{array}{l}\text { c) rollstuhlabhängig für } \\
\text { längere Strecken, } \\
\text { bedingt gehfähig }\end{array}$ & $\begin{array}{l}\text { - Stützapparate ohne } \\
\text { Kniesperre } \\
\text { - evtl. Fußhebehilfen } \\
\text { - Unterarmstützen } \\
\text { - mech. RST }\end{array}$ \\
\hline $\begin{array}{l}\text { L 5/S } 1 \\
\text { (M. triceps surae } \\
\text { M. flexores dig.) }\end{array}$ & c) gehfähig & $\begin{array}{l}\text { - ggf. Gehstöcke } \\
\text { - ggf. RST für Sport- } \\
\text { zwecke }\end{array}$ \\
\hline
\end{tabular}

Merke: bei kompletter Querschnittlähmung besteht grundsätzlich Blasen- und Mastdarmlähmung

(Quelle: Niethard FU, Pfeil ], Biberthaler P. Duale Reihe - Orthopädie und Unfallchirurgie. 8. Aufl. Thieme, Stuttgart 2017; Abb. B-8.18) 